\title{
Landau-Zener-Stückelberg Interferometry of a Single Electronic Spin in a Noisy Environment
}

\author{
Pu Huang, Jingwei Zhou, Fang Fang, Xi Kong, Xiangkun Xu, Chenyong Ju, ${ }^{*}$ and Jiangfeng $\mathrm{Du}^{\dagger}$ \\ Hefei National Laboratory for Physics Sciences at Microscale and Department of Modern Physics, \\ University of Science and Technology of China, Hefei, 230026, China
}

(Received 7 June 2011; published 8 August 2011)

\begin{abstract}
We demonstrate quantum coherent control of single electronic spins in a nitron-vacancy center in diamond by exploiting and implementing the general concept of Landau-Zener-Stückelberg interferometry at room temperature. The interferometry manipulates an effective two-level system of electronic spins which are coupled to the nearby ${ }^{14} \mathrm{~N}$ nuclear spin in the nitron-vacancy center as well as the nuclear spin bath in the diamond. With a microwave field to control the energy gap between the two levels and an AC field as the time-dependent driving field in Landau-Zener-Stïckelberg interferometry, the interference pattern can be generated and controlled by controlling a number of parameters in the fields, corresponding to coherent control of the state of the electronic spins. In particular, the interference pattern is observed oscillating as a function of the frequency of the microwave field. Decays in the visibility of the interference pattern are also observed and well explained by numerical simulation which takes into account the thermal fluctuations arising from the nuclear bath. Therefore, our work also demonstrates that Landau-Zener-Stückelberg interferometry can be used for probing decoherence processes of electronic spins.
\end{abstract}

DOI: 10.1103/PhysRevX.1.011003

Subject Areas: Photonics, Quantum Information, Spintronics

Landau-Zener (LZ) tunneling is a well-known phenomenon associated with strong driving fields. When a two-level system is driven through the avoided level crossing, LZ tunneling can be controlled in such a way to create various superpositions of the energy eigenstates, analogous to a coherent beam splitter. Combining two consecutive LZ tunneling events leads to Landau-Zener-Stückelberg (LZS) quantum interference, which is analogous to the MachZehnder (MZ) interferometry[1]. LZS interference has been observed in Rydberg atoms [2,3], quantum point contacts [4], and, recently, in mesoscopic superconducting Josephson devices [1,5], ultracold molecular gases[6], and optical lattices [7]. LZ tunneling and LZS interference have also been exploited for quantum-state preparation [8,9] and manipulation [10-12]. Interaction of a quantum state with the environment disturbs the coherence of the quantum system and therefore manifests in the LZS interference pattern [13-15]. LZS interferometry thus provides crucial information on the environment-induced decoherence process.

Single electronic spins of the nitrogen-vacancy (NV) center have been one of the most popular candidates for a qubit. Their high controllability and favorable coherence times hold promise for room-temperature quantum information processing [16-19] and highly sensitive

\footnotetext{
*cyju@ustc.edu.cn
}

${ }^{\dagger}$ djf@ustc.edu.cn

Published by the American Physical Society under the terms of the Creative Commons Attribution 3.0 License. Further distribution of this work must maintain attribution to the author(s) and the published article's title, journal citation, and DOI. magnetometry [20-22]. Initialization of a NV-center spin into the desired pure state can be done via optical pumping with high efficiency. Quantum coherent controls of the individual spin can be realized using the conventional microwave pulse controls. A NV center is also an ideal platform for studying quantum phenomena, especially the processes in a stong-driving-field regime such as anharmonic dynamics [23] and multifrequency spectra [24].

In this article, we carried out LZS interferometry on a $\mathrm{NV}$ center in high-purity diamond and demonstrated the feasibility of using LZS interferometry for quantum coherent control of single electronic spins. In this experiment, we first create, for electronic spin states, the analogue of a coherent beam splitter for optical beams based on the LZ tunneling process. This is experimentally realized by a driving field assisted by a microwave field. By repeating this process twice under different microwave frequencies, the quantum interference known as the Stückelberg oscillation is observed. The decay in the interference fringes agrees well with numerical simulations, which take into account the hyperfine coupling of the electronic spin with the surrounding nuclear spin bath. Our study shows that the thermal fluctuation of the nuclear spins is the dominant cause of the observed loss of coherence at room temperature.

LZ tunneling was first studied by Landau and Zener $[25,26]$. A description of the variety of phenomena related to LZ tunneling and LZS interference can be found in a recent review [27]. The problem relies on a two-level system, described by the LZ Hamiltonian,

$$
H_{\mathrm{LZ}}=-\frac{\Delta}{2} \sigma_{x}-\frac{\varepsilon(t)-\varepsilon_{0}}{2} \sigma_{z}
$$


which contains the Pauli matrices $\sigma_{x}$ and $\sigma_{z}$, a minimum energy separation $\Delta$ (the avoided level crossing), a time-dependent driving field $\varepsilon(t)$, and an offset $\varepsilon_{0}$. To implement LZ tunneling, the system is set initially in an eigenstate of $\sigma_{z}$, denoted by $|0\rangle$, while the magnitude of the oscillating driving field $\varepsilon(t)$ is set to be much larger than $\Delta$. In this way, the initial state $|0\rangle$ is close to one of the eigenstates of $H_{\mathrm{LZ}}$. Next, $\varepsilon(t)$ is gradually turned down, and as the system is swept through the avoided level crossing where $\varepsilon\left(t_{1}\right)=\varepsilon_{0}$, it undergoes LZ tunneling and its state splits from $|0\rangle$ into a superposition of $|0\rangle$ and $|1\rangle$. The probability of the system remaining in $|0\rangle$ is given by the well-known LZ formula:

$$
P_{\mathrm{T}}=\exp \left(-\frac{\pi}{2} \delta\right)
$$

where $\delta=\Delta^{2} / v$ and $v$ is the sweep velocity, which is equal to the value of $d \varepsilon(t) / d t$ at the avoided crossing. The system subsequently evolves and its decompositions into $|0\rangle$ and $|1\rangle$ accumulate a relative phase. When $\varepsilon(t)$ is swept back to the avoided level crossing at $t_{2}$, quantum interference occurs. The relative phase $\theta_{12}$ is given by

$$
\theta_{12}=\int_{t_{1}}^{t_{2}} E_{01}(t) d t
$$

with $E_{01}(t)$ the energy difference between $|0\rangle$ and $|1\rangle$. It is $\theta_{12}$ that gives rise to the interference fringes in the occupation probability known as Stückelberg oscillations. The LZS interferometer can also be conceived as a MZ interferometer, as shown in Fig. 1(b).

The key issue in LZ tunneling is the realization of an avoided level crossing in energy states. For electronic spins in the NV center, an avoided level cross cannot be realized directly by electron spin resonance, where a microwave field of formidable strength would be required due to the large crystal splitting of the NV center. In our scheme, the avoided level crossing is realized in the rotating frame where a microwave field can act as the minimum energy separation $[23,24,28]$. We consider the NV-center system, which includes electronic spins in a triple state and a nearby ${ }^{14} \mathrm{~N}$ nuclear spin. The Hamiltonian can be written as

$$
H_{\mathrm{NV}}=D S_{z}^{2}+g_{e} \mu_{B} B_{z} S_{z}+A_{z} I_{z} S_{z}
$$

Here, $D \approx 2.87 \mathrm{GHz}$ is the crystal field splitting and $B_{z}$ is the external magnetic field applied along the $z$ axis ([111] crystal axis), which lifts the degeneracy of the electronic spin states $|+1\rangle_{e}$ and $|-1\rangle_{e} \cdot \mu_{B}$ is the Bohr magneton and $g_{e}$ is the electron $g$ factor. The third term of Eq. (4) is the hyperfine coupling between the electronic spin and the ${ }^{14} \mathrm{~N}$ nuclear $\operatorname{spin}(A \approx 2.18 \mathrm{MHz})$. To realize the corresponding avoided level crossing in such a composite spin system, we focus on the subspace spanned by $|0\rangle_{e}|0\rangle_{I}$ and $|+1\rangle_{e}|0\rangle_{I}$ as in Fig. 1(c), which will be denoted by $|0\rangle$ and $|1\rangle$ in the following. Applying a microwave field $\Delta \cos \left(\omega_{\mathrm{MW}} t\right)$ along the $x$ axis selectively excites the
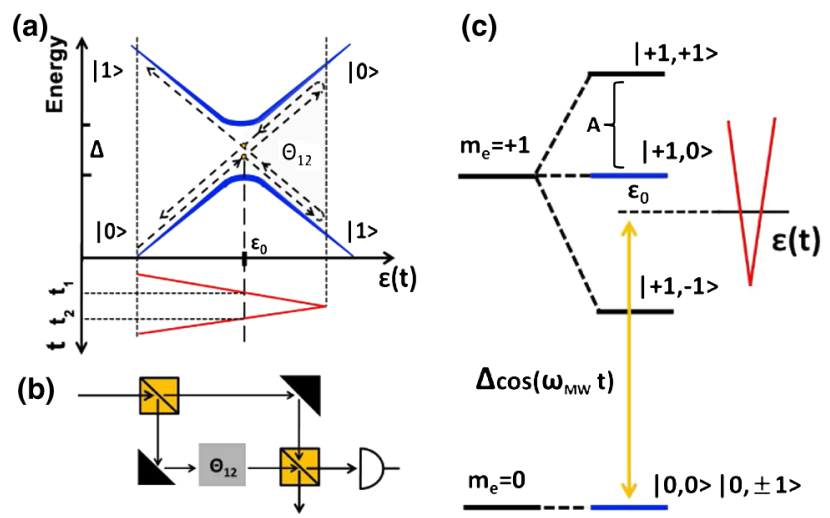

FIG. 1. Scheme for realizing a LZS interferometer on a single spin of the NV center in diamond. (a) Energy diagram of a twolevel LZS interferometer: starting at $|0\rangle$, the system is driven through the avoided level crossing (at $t_{1}$ ) and splits into a superposition of $|0\rangle$ and $|1\rangle$ via LZ tunneling. After accumulating a relative phase $\theta_{12}$, the system evolves back to the avoided level crossing (at $t_{2}$ ) where quantum interference occurs. (b) $\mathrm{A} \mathrm{MZ}$ interferometer can be used to describe the LZS interferometer, with LZ tunneling acting as an optical beam splitter. (c) Realization of the LZ Hamiltonian in a NV center: the two-level system of the LZS interferometer is expanded in electronic spin states $m_{e}=0$ and $m_{e}=+1$ with the ${ }^{14} \mathrm{~N}$ nuclear spin in the $m_{I}=0$ state, respectively, (blue lines). A selective microwave field with frequency $\omega_{\mathrm{MW}}$ (yellow double arrow) and with strength $\Delta$ can act as the minimum energy separation after going into the rotating frame; $\varepsilon_{0}$ in the LZ problem can be tuned directly by the microwave frequency. The driving field $\varepsilon(t)$ (red folded lines) is applied along the [111] crystal axis.

transition $|0\rangle \leftrightarrow|1\rangle$ when $\Delta \ll A$. In the rotating frame with the microwave frequency, $\Delta$ acts as a static field along the $x$ axis. Finally, by applying a time-dependent field $\varepsilon(t)$ along the $z$ axis with an amplitude smaller than $A_{z}$, the dynamics of $|0\rangle$ and $|1\rangle$ in the rotating frame is determined by $H_{\mathrm{LZ}}$. By tuning the strength of the microwave field, one can change the minimum energy separation $\Delta$, while by tuning the microwave frequency, $\varepsilon_{0}$ can be controlled.

We now describe the experimental setup for the demonstration of the LZS interferometry. The experiment is carried out on a home-built confocal microscope operated at room temperature. The sample is a type IIa single crystal diamond with an abundance of nitrogen electron spins less than $5 \mathrm{ppb}$. A single NV center is investigated via a microscope mounted on a piezoscanner by its fluorescence signals. A Hanbury-Brown-Twiss setup with two photodetectors is used to ensure a single NV is selected (data not shown). A $532 \mathrm{~nm}$ laser is used to initialize and read out the system. To manipulate the electronic spin coherently, a microwave signal is first generated by a ratio signal generator, and then a linear amplifier is employed to enhance the microwave power output. Finally, a $20 \mu \mathrm{m}$ diameter copper wire terminated by a $50 \Omega$ resistance is used to radiate the microwave field to the NV center. The degeneracy between $|+1\rangle_{e}$ and $|-1\rangle_{e}$ is lifted by an external 
magnetic field generated by three pairs of Helmholtz coils, with resolution $\approx 0.5 \mathrm{G}$ in magnitude and $5^{\circ}$ in direction. In the experiment, a magnetic field of $5 \mathrm{G}$ is employed. The driving field $\varepsilon(t)$ in $H_{\mathrm{LZ}}$ is generated by an arbitrary waveform generator (AWG) and the signal is directly sent to the sample via the copper wire. The typical frequency of the driving field is several $\mathrm{kHz}$, which is much lower than the microwave frequency, and thus only the components along the $z$ axis contribute. All signals are synchronized by a pulse generator. To build up sufficient statistics, we use typically $10^{5}$ cycles in a single measurement. $\Delta$ is calculated by using the output power of the amplifier and the amplitude of the driving field from the output voltage of the AWG. Typically, $\Delta \approx 500 \mathrm{kHz}$ at $20 \mathrm{dBm}$ output, while $4 \mathrm{~V}$ (peak-to-peak value of the sine wave) in an AWG corresponds to a driving field amplitude of $1.4 \mathrm{MHz}$.

Next, we describe the demonstration of the LZS interferometer. As the first step, we implement LZ tunneling that acts as a "beam splitter" for the (electronic) spin state of the system. Figure 2 shows the measured and simulated LZ tunneling dynamics under a driving field $\varepsilon \cos (2 \pi \omega t)$ for different values of the adiabaticity parameter $\delta=$ $\Delta^{2} / \varepsilon \omega$. The simulation is based on $H_{\mathrm{LZ}}$ and the environment is not taken into account. By tuning the adiabaticity parameter, the tunneling probability can be controlled. Such a beam splitter can be further exploited to serve as

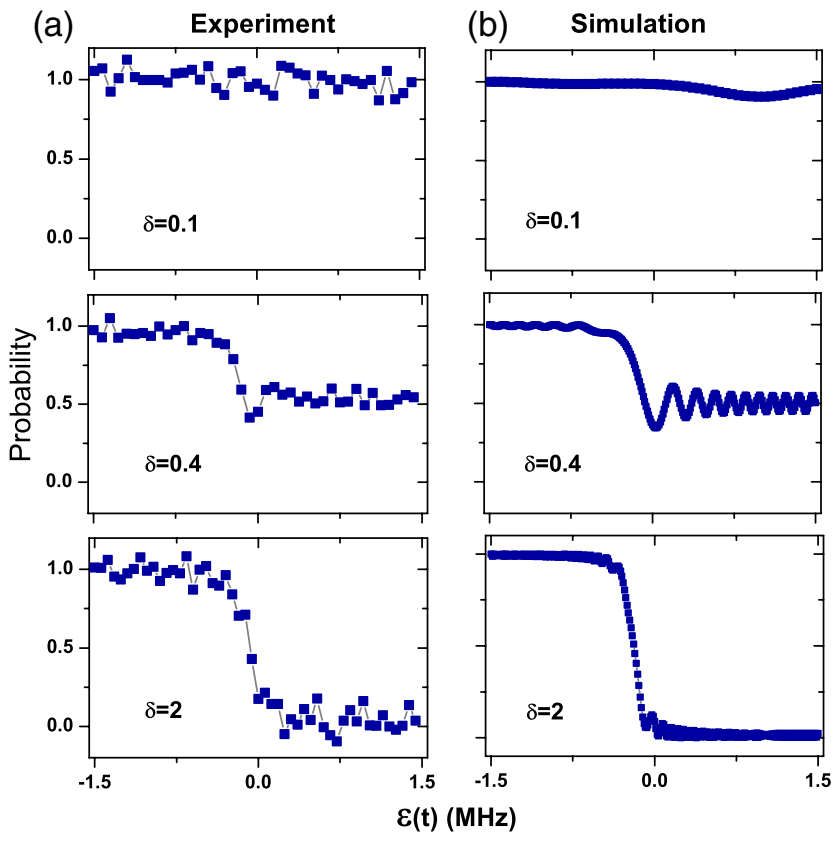

FIG. 2. Dynamics of the LZ tunneling. Measured (left) and simulated (right) dynamics of LZ tunneling under the driving field $\varepsilon(t)=\varepsilon \cos (2 \pi \omega t)$ for different values of the adiabaticity parameter $\delta=\Delta^{2} / \varepsilon \omega$. To measure this curve, we set $\varepsilon=$ $1.5 \mathrm{MHz}$ and $\Delta=0.11 \mathrm{kHz}$ and used different $\omega$. For the case $\delta \ll 1$, the system remains in $|0\rangle$, while for $\delta \gg 1$, the system evolves adiabatically with the driving field. the LZS interferometer. According to classical LZS interference theory [27], for a system that begins in $|0\rangle$, the probability $P$ of the system staying in the same state after undergoing two consecutive LZ tunneling events is given by

$$
P=4 P_{\mathrm{T}}\left(1-P_{\mathrm{T}}\right) \sin (\Phi)^{2} .
$$

Here, $P_{\mathrm{T}}$ is the LZ tunneling rate, and $\Phi=\Phi_{S}+\theta_{12}$, with $\Phi_{S}$ coming from the Stokes phase. Figure 3(a) shows the characteristic dynamics of the LZS interference process, while $P_{\mathrm{T}}$ as a function of sweeping velocity $v$ is plotted in Fig. 3(b). To extract the quantum coherence term $\sin (\Phi)^{2}$ in Eq. (5), we defined the interference visibility $V$ as $V=$ $P / 4 P_{\mathrm{T}}\left(1-P_{\mathrm{T}}\right)$. Without noises, $V$ is expected to oscillate between 0 and 1 as a function of $\theta_{12}$. However, as shown by the observed Stückelberg oscillation [Fig. 3(b)], the
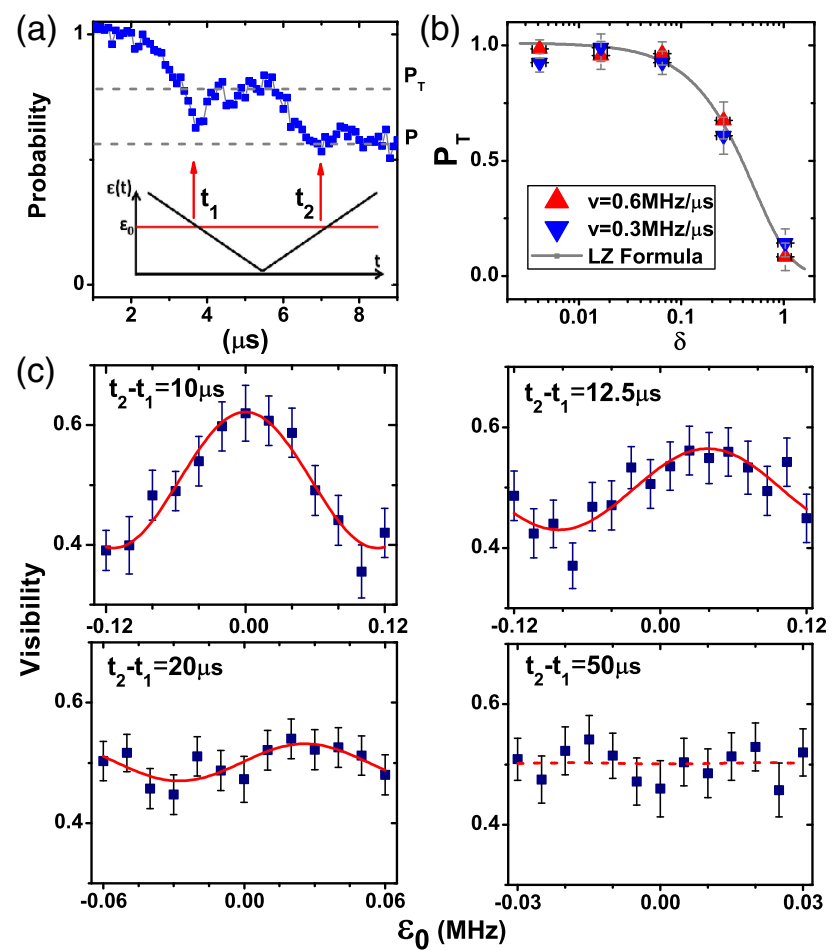

FIG. 3. LZS interferometer. (a) Dynamics of the LZS interference process driven by $\varepsilon(t)=v t$ (inset). Gray lines indicate the probability of being in the state $|0\rangle$ after passing through the first $\left(P_{\mathrm{T}}\right)$ and the second avoided level crossing $(P)$. (b) $P_{\mathrm{T}}$ as a function of the adiabaticity parameter $\delta$ (triangles) for different sweeping velocities. The result is in good agreement with the LZ formula (2). (c) Measured Stückelberg oscillation curves (blue squares) for different evolution durations $t_{2}-t_{1}$. The red line represents a fit to a cosine function in order to obtain the visibility. The dashed line in the last subfigure is a guide for the eyes since the visibility is nearly zero. In measuring this curve, we have adjusted the microwave power to bring $P_{\mathrm{T}}$ close to $1 / 2$, so that the oscillation amplitude is a maximum according to the classical LZ theory. The measured visibility is defined as $P / 4 P_{\mathrm{T}}\left(1-P_{\mathrm{T}}\right)$. 
visibility of interference fringes decreases as the duration of the interference process increases. Such an effect clearly indicates a loss of quantum coherence between $|0\rangle$ and $|1\rangle$.

To understand the observed decay in the interference pattern, the environment must be taken into account. In high-purity single crystals, where the abundance of nitrogen electron spins is less than $5 \mathrm{ppb}$, the main source of decoherence comes from the dipolar interaction of the electronic spin with surrounding ${ }^{13} \mathrm{C}$ nuclear spins [29]. This interaction, together with the dynamics of the nuclear spin bath, can be expressed by $\hat{b}_{z}|1\rangle\langle 1|+H_{\text {bath }}$ in the state subspace of the LZS interferometer. Here $\hat{b}_{z}$ is the coupling to the nuclear spin bath, which can be written as $\Sigma_{j} \mathbf{A}_{j} \cdot \mathbf{I}_{j}$, with $\mathbf{A}_{j}$ the coupling coefficient for the $j$-th nuclear spin $\mathbf{I}_{j}$ (the coupling strength is of the order of $\mathrm{kHz}$ ). The fluctuation perpendicular to the $z$ axis is negligible, since it is too weak to cause the spin-flip relaxation. $H_{\text {bath }}$ describes the dynamics of the bath, which includes the Zeeman splitting (several kHz) of the nuclear spins in the external magnetic field and the dipolar interaction between nuclear spins (of the order of $\mathrm{Hz}$ ). During the interference process, which occurs within tens of microseconds, the dynamics of the bath are negligible. We therefore expect that only the statistical fluctuations arising from the random orientations of the ${ }^{13} \mathrm{C}$ nuclear spins at room temperature contribute to the interference process. These fluctuations follow a Gaussian distribution $\exp \left(-b_{z}^{2} / 2 \beta^{2}\right)$ [30], where $\beta$ can be directly extracted from the free induction decay (FID) measurement [Fig. 4(b)]. It is found that $\beta=0.056 \mathrm{kHz}$ for the NV center under study.

Based on these considerations, numerical simulations were performed. The measured and simulated results are shown in Fig. 4(a). Good agreement between experiment and theory clearly establishes the decay mechanism as being due to nuclear spins. One can also capture the essence of the observations through the intuitive picture presented in Fig. 4(c). The phase giving rise to the interference fringes comes from the energy accumulated between two LZ tunneling points and is proportional to the duration of the interference process multiplied by the amplitude of the driving field. The presence of the effective field $b$ can change the position of the avoided level crossing and therefore cause fluctuations in $\theta_{12}$. As a result, the oscillations are washed out and the visibility decreases. This effect becomes more significant as the duration of the total process increases.

In conclusion, we have demonstrated a "beam splitter" for the electronic spin states of the NV center at room temperature based on LZ tunneling. Our results showed that the tunneling probability is only given by the adiabaticity parameter $\delta$, which agrees with the original prediction of the LZ formula. By combining two such beam splitters, the LZS interferometer is realized and the Stuickelberg oscillation is observed. The decay in the visibility of the interference fringes at room temperature is
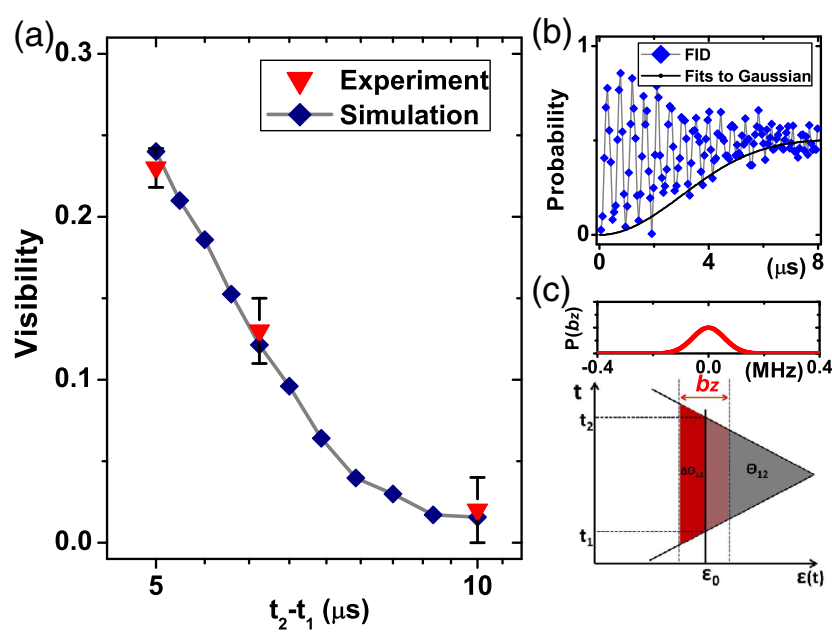

FIG. 4. Decoherence in the LZS process. (a) Measured (red triangles) and simulated (blue rectangles) visibility as a function of duration between the two LZ tunneling events. Agreement between theory and experiment confirms that the decay of the LZS visibility is dominated by the same source that results in the FID decay, which is generally the thermal fluctuations of the nuclear spin bath. (b) Measured FID signal; data are fitted to Gaussian decay (black line). (c) An intuitive picture can be employed to understand these observations. The fluctuations of nuclear spins produce an effective magnetic field $b_{z}$. It changes $\theta_{12}$, which is proportional to the area of the gray regime, with the result that the phase information is washed out.

shown to be caused by the thermal fluctuation of nuclear spins and agrees well with numerical simulations. Our work establishes the feasibility of using LZS interferometry for quantum coherent control and for probing the decoherence processes of single spin in the NV center. Very recent research shows that the LZ process can be used to realize in a NV center a coherent state exchange between the electronic spin and the nearby nitrogen nuclear spin, which can be exploited as a quantum memory [31]. We anticipate that more interesting applications will be applied to NV centers based on LZS interferometry.

We thank W. Yao and D. Culcer for their helpful discussions and for reading the paper. This work was supported by the National Natural Science Foundation of China (Grant No. 91021005), the CAS, and the National Fundamental Research Program (Grant No. 2007CB925200).

[1] W. D. Oliver, Y. Yu, J.C. Lee, K. K. Berggren, L. S. Levitov, and T. P. Orlando, Mach-Zehnder Interferometry in a Strongly Driven Superconducting Qubit, Science 310, 1653 (2005).

[2] S. Yoakum, L. Sirko, and P. M. Koch, Stueckelberg Oscillations in the Multiphoton Excitation of Helium Rydberg Atoms: Observation With a Pulse of Coherent Field and Suppression by Additive Noise, Phys. Rev. Lett. 69, 1919 (1992). 
[3] M.C. Baruch and T.F. Gallagher, Ramsey Interference Fringes in Single Pulse Microwave Multiphoton Transitions, Phys. Rev. Lett. 68, 3515 (1992).

[4] L. Y. Gorelik, N. I. Lundin, V. S. Shumeiko, R. I. Shekhter, and M. Jonson, Superconducting Single-Mode Contact as a Microwave-Activated Quantum Interferometer, Phys. Rev. Lett. 81, 2538 (1998).

[5] M. Sillanpää, T. Lehtinen, A. Paila, Y. Makhlin, and P. Hakonen, Continuous-Time Monitoring of Landau-Zener Interference in a Cooper-Pair Box, Phys. Rev. Lett. 96, 187002 (2006).

[6] M. Mark, T. Kraemer, P. Waldburger, J. Herbig, C. Chin, H.-C. Nägerl, and R. Grimm, Stückelberg Interferometry” with Ultracold Molecules, Phys. Rev. Lett. 99, 113201 (2007).

[7] Sebastian Kling, Tobias Salger, Christopher Grossert, and Martin Weitz, Atomic Bloch-Zener Oscillations and Stückelberg Interferometry in Optical Lattices, Phys. Rev. Lett. 105, 215301 (2010).

[8] D. J. Reilly, J. M. Taylor, J. R. Petta, C. M. Marcus, M. P. Hanson, and A.C. Gossard, Suppressing Spin Qubit Dephasing by Nuclear State Preparation, Science 321, 817 (2008).

[9] Hugo Ribeiro and Guido Burkard, Nuclear State Preparation via Landau-Zener-Stückelberg Transitions in Double Quantum Dots, Phys. Rev. Lett. 102, 216802 (2009).

[10] J. R. Petta, H. Lu, and A.C. Gossard, A Coherent Beam Splitter for Electronic Spin States, Science 327, 669 (2010).

[11] Guozhu Sun, Xueda Wen, Bo Mao, Jian Chen, Yang Yu, Peiheng Wu, and Siyuan Han, Tunable Quantum Beam Splitters for Coherent Manipulation of a Solid-State Tripartite Qubit System, Nature Commun. 1, 51 (2010).

[12] H. Ribeiro, J. R. Petta, and G. Burkard, "Harnessing the GaAs Quantum Dot Nuclear Spin Bath for Quantum Control, Phys. Rev. B 82, 115445 (2010).

[13] D. M. Berns, W. D. Oliver, S. O. Valenzuela, A. V. Shytov, K. K. Berggren, L. S. Levitov, and T. P. Orlando, Coherent Quasiclassical Dynamics of a Persistent Current Qubit, Phys. Rev. Lett. 97, 150502 (2006).

[14] M. S. Rudner, A. V. Shytov, L. S. Levitov, D. M. Berns, W. D. Oliver, S.O. Valenzuela, and T.P. Orlando, Quantum Phase Tomography of a Strongly Driven Qubit, Phys. Rev. Lett. 101, 190502 (2008).

[15] L. Du, M. Wang, and Y. Yu, Landau-Zener-Stückelberg Interferometry in the Presence of Quantum Noise, Phys. Rev. B 82, 045128 (2010).

[16] M. V. Gurudev Dutt, L. Childress, L. Jiang, E. Togan, J. Maze, F. Jelezko, A. S. Zibrov, P. R. Hemmer, and M. D. Lukin, Quantum Register Based on Individual Electronic and Nuclear Spin Qubits in Diamond, Science 316, 1312 (2007).
[17] P. Neumann, N. Mizuochi, F. Rempp, P. Hemmer, H. Watanabe, S. Yamasaki, V. Jacques, T. Gaebel, F. Jelezko, and J. Wrachtrup, Multipartite Entanglement Among Single Spins in Diamond, Science 320, 1326 (2008).

[18] P. Neumann, J. Beck, M. Steiner, F. Rempp, H. Fedder, P. R. Hemmer, J. Wrachtrup, and F. Jelezko, Single-Shot Readout of a Single Nuclear Spin, Science 329, 542 (2010).

[19] G. Balasubramanian et al., Ultralong Spin Coherence Time in Isotopically Engineered Diamond, Nature Mater. 8, 383 (2009).

[20] J. R. Maze et al., Nanoscale Magnetic Sensing with an Individual Electronic Spin in Diamond, Nature (London) 455, 644 (2008).

[21] G. Balasubramanian et al., Nanoscale Imaging Magnetometry with Diamond Spins Under Ambient Conditions, Nature (London) 455, 648 (2008).

[22] G. de Lange, D. Riste, V. V. Dobrovitski, and R. Hanson, Single-Spin Magnetometry with Multipulse Sensing Sequences, Phys. Rev. Lett. 106, 080802 (2011).

[23] G. D. Fuchs, V. V. Dobrovitski, D. M. Toyli, F. J. Heremans, and D. D. Awschalom, Gigahertz Dynamics of a Strongly Driven Single Quantum Spin, Science 326, 1520 (2009).

[24] L. Childress and J. McIntyre, Multifrequency Spin Resonance in Diamond, Phys. Rev. A 82, 033839 (2010).

[25] L. D. Landau, On the Theory of Transfer of Energy at Collisions II, Phys. Z. Sowjetunion 2, 46 (1932).

[26] Clarence Zener, Non-Adiabatic Crossing of Energy Levels, Proc. R. Soc. A 137, 696 (1932).

[27] S. Shevchenko, S. Ashhab, and F. Nori, Landau-ZenerStückelberg Interferometry, Phys. Rep. 492, 1 (2010).

[28] G. Sun, X. Wen, B. Mao, Y. Yu, J. Chen, W. Xu, L. Kang, P. Wu, and S. Han, Landau-Zener-Stückelberg Interference of Microwave-Dressed States of a Superconducting Phase Qubit, Phys. Rev. B 83, 180507 (R) (2011).

[29] L. Childress, M. V. Gurudev Dutt, J. M. Taylor, A. S. Zibrov, F. Jelezko, J. Wrachtrup, P. R. Hemmer, and M. D. Lukin, Coherent Dynamics of Coupled Electron and Nuclear Spin Qubits in Diamond, Science 314, 281 (2006).

[30] R. Hanson, V. V. Dobrovitski, A. E. Feiguin, O. Gywat, and D.D. Awschalom, Coherent Dynamics of a Single Spin Interacting with an Adjustable Spin Bath, Science 320, 352 (2008).

[31] G. D. Fuchs, G. Burkard, P. V. Klimov, and D. D. Awschalom, A Quantum Memory Intrinsic to Single Nitrogen-Vacancy Centres in Diamond, Nature Phys., advance online publication (doi:10.1038/nphys2026). 